\title{
STUDY ON DYNAMIC DAMAGE CAUSES OF SINGLE LAYER LATTICED SHELLS BASED ON REFINED MEMBER CALCULATION MODEL
}

\author{
Yang Ding ${ }^{1}$, Lin $\mathrm{Qi}^{2, *}$ and Zhongxian $\mathrm{Li}^{3}$ \\ ${ }^{1}$ Professor, Department of Civil Engineering, Tianjin University, Tianjin, China \\ ${ }^{2}$ Ph. $d$ candidate, Department of Civil Engineering, Tianjin University, Tianjin, China \\ ${ }^{3}$ Professor, Department of Civil Engineering, Tianjin University, Tianjin, China \\ *(Corresponding author: E-mail: qilin1208@163.com)
}

Received: 25 December 2010; Revised: 7 April 2011; Accepted: 12 April 2011

\begin{abstract}
Based on mechanical characteristics of the members of single layer latticed shells, two buckling types of structural compression members are presented. The pre-buckling and post-buckling mechanical behaviors of the structural member are simulated by different models, and the refined member calculation model is established. In this model the second order effect of the member is considered, and the possible buckle-straighten processes of the members and the form-disappear processes of the plastic hinges of the structure under earthquakes can be simulated. Numerical structure models of commonly used single layer latticed shells are created to analyze the causes of structural dynamic damages under earthquakes. The calculation results indicate that dynamic damage causes of single layer latticed shells vary with different structural types: dynamic damages of single layer spherical latticed shells are caused by buckling members which weaken the structural bearing capacity; dynamic damages of single layer cylindrical latticed shells are caused by concentrative plastic hinges in the end sections of some members which make portions of the structure turn to mechanisms; dynamic damages of single layer elliptic paraboloid latticed shells are caused by combined actions of the two causes mentioned above.
\end{abstract}

Keywords: Single layer latticed shell, Refined member calculation model, Member buckling, Plastic hinge, Second order effect, Dynamic damage cause

\section{INTRODUCTION}

The calculation method for single layer latticed shells is becoming a major research subject recently. Kani and Heidari [1] made an attempt to automatically calculate the bifurcation of the buckling path of shallow latticed domes using two-stage analysis of the space structure without introducing any geometrical imperfections. Yamada et al. [2] studied the effects of geometric imperfections on the nonlinear buckling behavior of rigidly jointed single-layer latticed domes under vertical loading. Hearn and Adams [3] established a method which used static joint equilibrium equations to determine a minimum set of adjustments to latticed geometry needed to achieve equilibrium reference states for shape selection for latticed structures with members that carry axial force only. Morris [4] presented a nonlinear elastic-plastic method for three dimensional beam elements which was applied to investigate the imperfection-sensitivity of latticed domes made up of tubular members. Kani and McConnel [5] made a numerical and experimental study on the collapse and post collapse behavior of shallow latticed domes. Although there are many scholars making investigations of single layer latticed shells, causes of dynamic damages of the structure under earthquakes are rarely carried on. Mechanical characteristics of single layer latticed shells vary with different structure types, so the causes of structural dynamic damages are not the same. The single layer latticed shell is a typical spatial structure composed of a great many members by certain geometrical law. Subject to dynamic loads, the single layer latticed shell undergoes loading-unloading process repeatedly, which leads to the possible buckle-straighten processes of the structural members and the form-disappear processes of the plastic hinges. The mechanical behaviors of the members and the structural bearing capacity are changing continuously. The refined member calculation model which can simulate the continuous mechanical behavior changes of the member should be established, so the accurate damage processes of single layer latticed shells can be modeled. 
There are two main problems if members of single layer latticed shells are modeled by general beam element of the general finite element analysis program: (1) The accuracy of the elastic-plastic element stiffness matrixes. By general finite element analysis program, the material constitutive relationships of the Gauss integral sections of the general beam element are used to calculate the element stiffness matrix. The elastic element stiffness matrix can be calculated correctly by this arithmetic if the member stays elastic. It is the end section of the member, which is not the Gauss integral section that firstly turns into plastic state commonly. Thus when the end section turns into plastic state, the element stiffness matrix calculated by general finite element analysis program is still elastic. Because of different loading history, material elastic-plastic constitutive relationships of different sections of the member are not the same, so it is not rational to calculate elastic-plastic element stiffness matrix based on the material constitutive relationship of the Gauss integral sections. (2) The simulation of the buckling members. If one structural member is modeled by a single general beam element of general finite element analysis program, buckling of the members cannot be simulated, and the decrease of the member bearing capacity is caused by material yielding rather than member buckling. In this case the member can bear the load far over the buckling critical load and the member bearing capacity is seriously overestimated. Buckling of the member can be simulated if one member is divided into more than one general beam element of general finite element analysis program. However, the post-buckling behaviors of the member and the plastic hinges forming in the end section and the central section cannot be simulated. How many general beam elements of general finite element analysis program one structural member should be divided into is also difficult to be appropriately determined.

To study the dynamic damage causes of single layer latticed shells under earthquakes, calculation model of the structure that tallies with the actual situation should be created. In this paper, two buckling types of the compression members of single layer latticed shells are presented. The pre-buckling and post-buckling mechanical behaviors of the structural member are simulated by different models, and the refined member calculation model is established. By this model the second order effect of the member is considered, and the possible buckle-straighten processes of the member and the form-disappear processes of the plastic hinge and the consequent complex change processes of structural bearing capacity under earthquakes can be simulated clearly. Based on the refined member calculation model, structural models are created to study the causes of dynamic damages of typical single layer latticed shells under earthquakes.

\section{BUCKLING TYPES OF THE MEMBER}

There are two possible buckling types of the compression members of single layer latticed shells: (1) Buckling type I. The plastic hinge forms in the end section of the member, and slenderness ratio of the member gets bigger. This may cause the buckling of the member. The members of single layer latticed shells bear concentrated forces at the nodes only, so it is the end section of the member where the stress is biggest. The buckling critical condition may not be satisfied when the plastic zone forms in the end section of the member. The plastic zone develops along with the increment of the load. When the plastic hinge forms in the end of the member and makes the slenderness ratio turn larger, the buckling critical condition gets easier to be med. Even a small increment of the external load may make the member buckling. The plastic zone or plastic hinge then forms in the central section of the buckled member as a combined action of both the axial compression force and the additional bending moment. (2) Buckling type II. The member bears compression axial force plenty big and the buckling critical condition is met without the plastic hinge forming in the end section. The plastic zone or plastic hinge then forms in the central section of the buckled member as a combined action of both the compression axial force and the additional bending moment. 


\section{PLASTIC HINGE IN THE END SECTION OF THE MEMBER}

Influences to mechanical behavior of the structural member caused by plastic hinge forming in the end section can be illuminated by the cyclic loading experiment on cantilever member (Fan [6]). Hysteresis loop of the experiment is illustrated in Figure 1, and full-range behavior of the cantilever member subjected to once cycling loading is illustrated in Figure 2. The curve in Figure 2 can be divided into 10 segments: (1) $O-A$ is the elastic loading segment; (2) outboard fibers in the end section of the member yield at point $A$, and the total cross end section yields at point $B$ when plastic hinge forms; (3) material of the end section of the member enters its strengthening stage in segment $B-C$, and the stress of the end section reaches ultimate strength at point $C$; (4) in segment $C-D$ the load that the member can bear decreases a little bit and the deformation becomes larger continuously; (5) $D-E$ is the unloading segment, and the plastic hinge in the end section of the member disappears; (6) $E-F$ is elastic loading segment; (7) outboard fibers in the end section of the member yields at point $F$, and the total cross end section yields at point $G$ when plastic hinge forms once again; (8) material of the end section of the member enters its strengthening stage in segment $G-H$, and the stress of the end section reaches ultimate strength at point $H$; (9) in segment $H-I$ the load that the member can bear decreases a little bit and the deformation becomes larger continuously; (10) $I-J$ is the unloading segment, and the plastic hinge in the end section of the member disappears. The residual deformation remains when the unloading process is completed at point $J$.

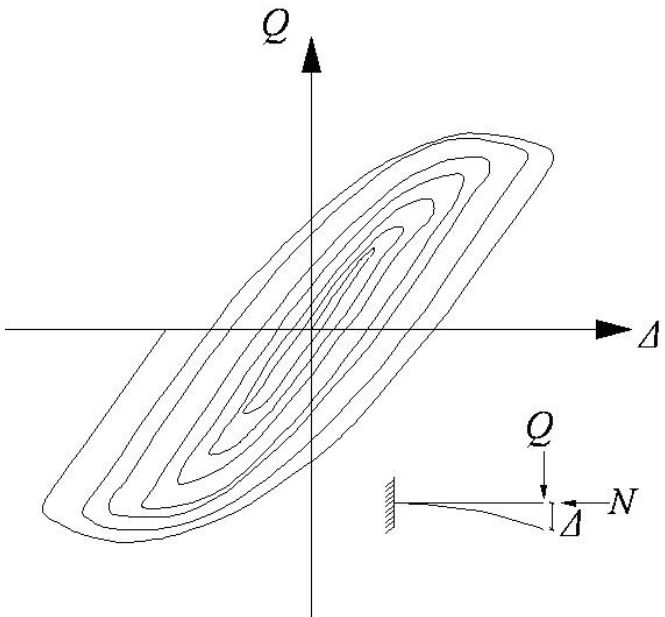

Figure 1. Hysteresis Loop of Cantilever

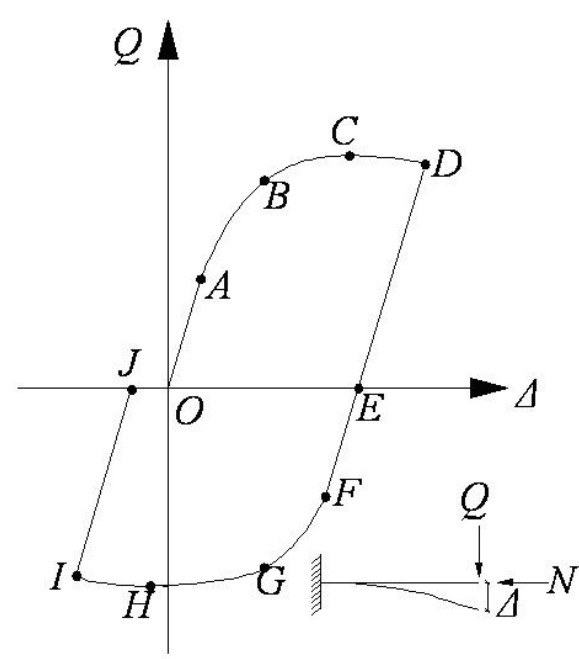

Figure 2. Full-range Behavior of Cantilever

\section{BUCKLING OF THE MEMBER}

\subsection{Influences to Mechanical Behavior of the Member Caused by Buckling}

Influences to mechanical behavior of the structural member caused by buckling can be illuminated by cyclic loading experiment on circular tube (Chen and Sugimoto [7]). Hysteresis loop of the experiment is illustrated in Figure 3, and full-range behavior of the circular tube subjected to once cycling loading is illustrated in Figure 4. The curve in Figure 4 can be divided into 9 segments: (1) $O-A$ is elastic loading segment; (2) the experimental specimen is circular tube with initial bending imperfection, thus the load-displacement curve in segment $A-B$ exhibits distinct nonlinearity. Buckling criteria is met at point $B$; (3) the bearing capacity of the member decreases in segment $B-C$, and the plastic hinge forms in the central section of the member as a combine action of both 
the axial compression force and the additional bending moment; (4) $C-D$ is the unloading segment; (5) $D-E$ is the elastic tension segment; (6) as the tension force increases, the plastic zone forms in the central section in segment $E-F$; (7) the member is nearly straightened in segment $F-G$, and the plastic hinge forms in the central section of the member once again as a combine action of both the axial compression force and the additional bending moment; (8) the member is completely straightened in segment $G-H$; (9) $H-I$ is the elastic unloading segment, and the residual deformation remains when unloading process is completed. When unloading occurs at point $G, G-I$ is the elastic unloading segment. It can be seen that mechanical behaviors of the member change a lot after the member buckles.

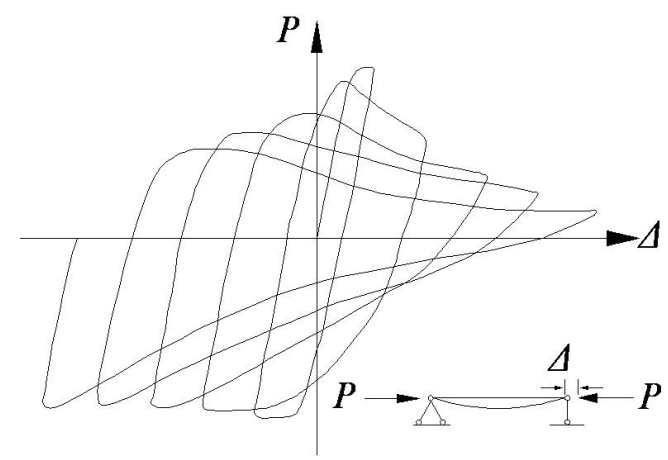

Figure 3. Hysteresis Loop of Circular Tube

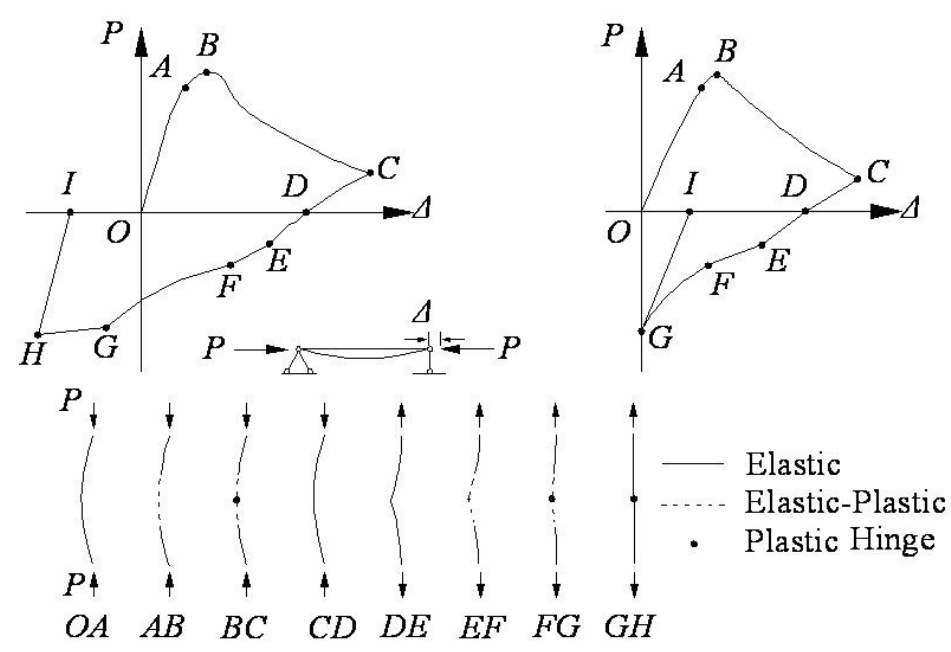

Figure 4. Full-range Behavior of Circular Tube

\subsection{Buckling Criteria for Circular Tube}

Members of single layer latticed shells, which bear spatial forces, are mainly circular tubes. According to a large number of experiments, the buckling criteria equation for circular tube bearing spatial forces is proposed by ISO (ISO [8]):

$$
I\left(f_{c}, f_{b 1}, f_{b 2}\right)=\frac{f_{c}}{F_{c}}+\frac{1}{F_{b}} \sqrt{\left(\frac{c_{m 1} f_{b 1}}{1-\frac{f_{c}}{F_{e 1}}}\right)^{2}+\left(\frac{c_{m 2} f_{b 2}}{1-\frac{f_{c}}{F_{e 2}}}\right)^{2}}
$$

Where $f_{c}=P / A$ is the axial compression stress, $P$ and $A$ are axial compression force and cross-sectional area respectively; $f_{b 1}$ and $f_{b 2}$ are the maximum bending stresses about the crosssection local 1 and 2 axes, and $f_{b 1}=M_{1} / W_{e}, f_{b 2}=M_{2} / W_{e}$, where $W_{e}$ is the section modulus of elasticity; $c_{m 1}$ and $c_{m 2}$ are reduction factors corresponding to the cross-section directions 1 and 2 respectively, and $c_{m 1}=c_{m 2}=0.85 ; F_{e 1}$ and $F_{e 2}$ are Euler buckling stresses corresponding to the local 1 or 2 direction, and $F_{e 1}=F_{y c} / \lambda_{1}^{2}, F_{e 2}=F_{y c} / \lambda_{2}^{2}, \lambda_{1}=k_{1} L_{1} / \pi i \sqrt{F_{y c} / E}, \lambda_{2}=k_{2} L_{2} / \pi i \sqrt{F_{y c} / E}$, where $L_{1}$ and $L_{2}$ are the unbraced lengths for the local 1 and 2 directions, $k_{1}$ and $k_{2}$ are the effective length factors in the local 1 and 2 directions, $i$ is the radius of gyration; $F_{c}$ and $F_{b}$ are the characteristic axial compressive stress and the characteristic bending stress respectively, where 


$$
\begin{aligned}
& F_{c}= \begin{cases}\left(1.0-0.28 \lambda^{2}\right) F_{y c} & \lambda \leq 1.34 \\
\frac{0.89282978}{\lambda^{2}} F_{y c} & \lambda>1.34\end{cases} \\
& F_{b}= \begin{cases}\frac{W_{p}}{W_{e}} \sigma_{s} & \frac{\sigma_{s} D}{E t} \leq 0.051 \\
\left(1.133386-2.58 \frac{\sigma_{s} D}{E t}\right) \frac{W_{p}}{W_{e}} \sigma_{s} & 0.0517<\frac{\sigma_{s} D}{E t} \leq 0.103 \\
\left(0.945198-0.76 \frac{\sigma_{s} D}{E t}\right) \frac{W_{p}}{W_{e}} \sigma_{s} & 0.1034<\frac{\sigma_{s} D}{E t} \leq \frac{120 \sigma_{s}}{E}\end{cases}
\end{aligned}
$$

Where $F_{y c}$ is the characteristic local buckling stress.

$$
F_{y c}= \begin{cases}\sigma_{s} & \frac{5 \sigma_{s} D}{3 E t} \leq 0.170 \\ \left(1.04654873-0.27381606 \frac{5 \sigma_{s} D}{3 E t}\right) \sigma_{s} & 0.170<\frac{5 \sigma_{s} D}{3 E t} \leq 1.911 \\ \frac{0.6 E t}{D} & \frac{5 \sigma_{s} D}{3 E t}>1.911\end{cases}
$$

Where $\sigma_{s}$ is the yield stress; $D$ is the outside diameter and $t$ is the thickness of tube wall; $\lambda=\max \left(\lambda_{1}, \lambda_{2}\right) ; W_{p}=\left[D^{3}-(D-2 t)^{3}\right] / 6$.

The member gets buckled when $I\left(f_{c}, f_{b 1}, f_{b 2}\right) \geq 1.0$. To prevent misjudgment in cases where negligible axial force exists with large bending moments, an additional inequality is used. This additional check, called the strength equation, takes the following form:

$$
S\left(f_{c}, f_{b 1}, f_{b 2}\right)=\frac{f_{c}}{F_{y c}}+\frac{1}{F_{b}} \sqrt{f_{b 1}^{2}+f_{b 2}^{2}}
$$

Thus the buckling criteria for circular tube bearing spatial forces is

$$
\left\{\begin{array}{l}
I\left(f_{c}, f_{b 1}, f_{b 2}\right) \geq 1.0 \\
S\left(f_{c}, f_{b 1}, f_{b 2}\right) \leq 1.0
\end{array}\right.
$$

It is the buckling critical state of the circular tube when $I\left(f_{c}, f_{b 1}, f_{b 2}\right)=1.0$ and $S\left(f_{c}, f_{b 1}, f_{b 2}\right) \leq 1.0$. The buckling critical axial force is

$$
P_{c r}=f_{c} A
$$




\section{MEMBER CALCULATION MODEL}

\subsection{Calculation Model of the Pre-buckling Member}

Displacement interpolation equations of the member considering second order effect are derived based on the deformed configuration. Deformed configuration of the member bearing axial tension force, shear force and bending moment is illustrated in Figure 5.

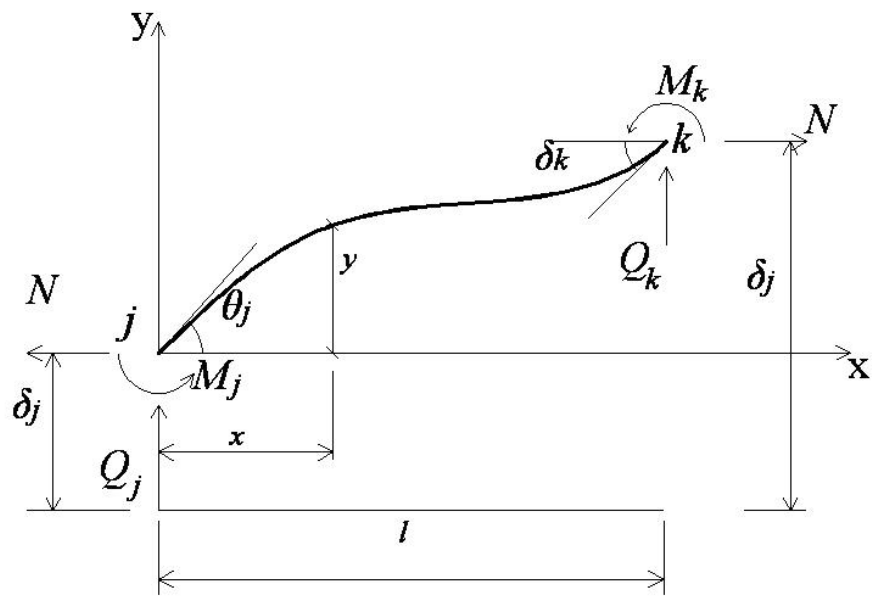

Figure 5. Deformed Configuration of the Member

Translational displacements caused by bending moment and shear force are $y_{\mathrm{M}}$ and $y_{\mathrm{Q}}$ respectively. $y_{\mathrm{M}}^{\prime \prime}=-M / E I$ and $y_{\mathrm{Q}}^{\prime}=-\mu Q /(G A)$, so second derivative of the total lateral displacement of arbitrary section of the member is given by the following equation:

$y^{\prime \prime}=y_{\mathrm{M}}^{\prime \prime}+y_{\mathrm{Q}}^{\prime \prime}=-\frac{M}{E I}-\frac{\mu}{G A} \cdot \frac{\mathrm{d} Q}{\mathrm{~d} x}=-\frac{M}{E I}=\frac{Q_{j}}{E I} x-\frac{M_{j}-N}{E I}$

Where $\mu$ is shear-shape coefficient; $E$ and $G$ are elasticity modulus and shear modulus of the material; $I$ is sectional inertia moment.

Differential equation of the total lateral displacement of arbitrary section of the member bearing compression axial force has the same expression as Eq. 8 except for the sign before variable $N$ which turns to be positive. Introducing boundary condition: $y=0$ when $x=0$ and $y=\delta_{k}-\delta_{j}$ when $x=l$, lateral displacement interpolation equations expressed by stability functions for both tension and compression members can be derived from the differential equations. Maclaurin series expansion is used to replace the stability functions and the uniform lateral displacement interpolation equations for both tension and compression members are given by the following equation:

$y=N_{1} \delta_{j}+N_{2} \theta_{j}+N_{3} \delta_{k}+N_{4} \delta_{k}$

Differential equation of the rotation displacement of arbitrary section of the member is given by the following equation: 
$\theta(x)=\frac{\mathrm{d} y}{\mathrm{~d} x}-\frac{\mu Q}{G A}$

Similarly, Maclaurin series expansion is used to replace the stability functions in the solution of Eq. 10 and the uniform rotation displacement interpolation equations for both tension and compression members are given by the following equation:

$\theta(x)=N_{5} \delta_{j}+N_{6} \theta_{j}+N_{7} \delta_{k}+N_{8} \delta_{k}$

Parameters in Eq. 10 and Eq. 11 are defined as follows:

$$
\begin{aligned}
& N_{1}=-\frac{1}{\varphi} \chi_{\mathrm{o}} \cdot \psi_{\mathrm{o}}+\frac{1}{\varphi} \rho \xi\left[1+\chi_{\mathrm{j}}\right]\left[1+\psi_{\mathrm{j}}\right]-\frac{\eta}{\varphi} \rho \xi\left[1+\chi_{\mathrm{j}}\right] \\
& N_{2}=-\frac{l \psi_{\mathrm{o}}}{\eta \varphi}\left[\left(1+\chi_{\mathrm{j}}\right)-\eta\left(1+\chi_{\mathrm{o}}\right)\right]-\frac{x}{\eta \varphi}\left[\chi_{\mathrm{o} p}-\rho_{0} \eta\left(1+\chi_{\mathrm{j}}\right)\right] \cdot\left(1+\psi_{\mathrm{j}}\right)-\frac{x}{\varphi} \chi_{\mathrm{o}} \\
& N_{3}=\frac{1}{\varphi} \chi_{\mathrm{o}} \psi_{\mathrm{o}}-\frac{1}{\varphi} \rho \xi\left(1+\chi_{\mathrm{j}}\right) \cdot\left(1+\psi_{\mathrm{j}}\right)+\frac{\eta \rho \xi}{\varphi}\left(1+\chi_{\mathrm{j}}\right) \\
& N_{4}=\frac{l \psi_{\mathrm{o}}}{\eta \varphi}\left[\eta-\left(1+\chi_{\mathrm{j}}\right)\right]+\frac{x}{\eta \varphi} \chi_{\mathrm{o}}\left(1+\psi_{\mathrm{j}}\right)-\frac{x}{\varphi} \chi_{\mathrm{o}} \\
& N_{5}=-\frac{\rho \xi}{l \varphi} \chi_{\mathrm{o}}\left(1+\psi_{\mathrm{j}}\right)+\frac{\rho}{l \varphi}\left(1+\chi_{\mathrm{j}}\right) \cdot\left(1+\psi_{\mathrm{o}}\right)-\frac{\rho \eta^{2}}{l \varphi}\left(1+\chi_{\mathrm{j}}\right) \\
& N_{6}=-\frac{\rho \xi}{\eta \varphi}\left[\left(1+\chi_{\mathrm{j}}\right)-\eta\left(1+\chi_{\mathrm{o}}\right)\right] \cdot\left(1+\psi_{\mathrm{j}}\right)+\frac{1}{\eta \varphi}\left[\frac{\eta \rho_{0}}{l^{2}}\left(1+\chi_{\mathrm{j}}\right)-\chi_{\mathrm{o}}\right] \cdot\left(1+\psi_{\mathrm{o}}\right)-\frac{\eta}{\varphi} \chi_{\mathrm{o}} \\
& N_{7}=\frac{\rho \xi}{l \varphi} \chi_{\mathrm{o}}\left(1+\psi_{\mathrm{j}}\right)-\frac{\rho}{l \varphi}\left(1+\chi_{\mathrm{j}}\right) \cdot\left(1+\psi_{\mathrm{o}}\right)+\frac{\rho \eta^{2}}{l \varphi}\left(1+\chi_{\mathrm{j}}\right) \\
& N_{8}=-\frac{\rho \xi}{\eta \varphi}\left(1-\eta+\chi_{\mathrm{j}}\right) \cdot\left(1+\psi_{\mathrm{j}}\right)+\frac{1}{\eta \varphi} \chi_{\mathrm{o}}\left(1+\psi_{\mathrm{o}}\right)-\frac{\eta}{\varphi} \chi_{\mathrm{o}}
\end{aligned}
$$

where $\eta=1+\mu N / G A ; \rho=|N| l^{2} / \eta E I ; \rho_{0}=N l^{2} / \eta E I ; \quad \chi_{\mathrm{o}}=\sum_{n=1}^{\infty} \rho^{n} /(2 n) ! ; \chi_{\mathrm{j}}=\sum_{n=1}^{\infty} \rho^{n} /(2 n+1) !$; $\psi_{\mathrm{o}}=\sum_{n=1}^{\infty}\left(\rho \xi^{2}\right)^{n} /(2 n) ! ; \quad \psi_{\mathrm{j}}=\sum_{n=1}^{\infty}\left(\rho \xi^{2}\right)^{n} /(2 n+1) ! ; \varphi=2-2\left(1+\chi_{\mathrm{o}}\right)+\rho \eta\left(1+\chi_{\mathrm{j}}\right) ; \quad \xi=x / l$.

Axial interpolation equations of the member are defined by linear equations. Extend the displacement interpolation equations of the planar member to three-dimensional space, and displacement interpolation equation matrix of the spatial member is given by the following equation: 
$\boldsymbol{u}_{0}=\boldsymbol{N} \boldsymbol{u}=\left[\begin{array}{cccccccccccc}1-x / l & 0 & 0 & 0 & 0 & 0 & x / l & 0 & 0 & 0 & 0 & 0 \\ 0 & N_{1} & 0 & 0 & 0 & N_{2} & 0 & N_{3} & 0 & 0 & 0 & N_{4} \\ 0 & 0 & N_{1} & 0 & -N_{2} & 0 & 0 & 0 & N_{3} & 0 & -N_{4} & 0 \\ 0 & 0 & 0 & 1-x / l & 0 & 0 & 0 & 0 & 0 & x / l & 0 & 0 \\ 0 & 0 & -N_{5} & 0 & N_{6} & 0 & 0 & 0 & -N_{7} & 0 & N_{8} & 0 \\ 0 & N_{5} & 0 & 0 & 0 & N_{6} & 0 & N_{7} & 0 & 0 & 0 & N_{8}\end{array}\right] \boldsymbol{u}=\left[\begin{array}{l}\boldsymbol{N}_{u x} \\ \boldsymbol{N}_{u \boldsymbol{u}} \\ \boldsymbol{N}_{u z} \\ \boldsymbol{N}_{\theta x} \\ \boldsymbol{N}_{\theta \boldsymbol{y}} \\ \boldsymbol{N}_{\theta z}\end{array}\right] \boldsymbol{u}$

Where $\boldsymbol{u}_{0}$ is the centroid displacement of arbitrary section of the member, and $\boldsymbol{u}_{0}=\left\{u_{x 0} u_{y 0} u_{z 0} \theta_{x 0} \theta_{y 0} \theta_{z 0}\right\} ; \boldsymbol{u}$ is the centroid displacement of the end section of the member, and $\boldsymbol{u}=\left\{u_{x j} u_{y j} u_{z j} \theta_{x j} \theta_{y j} \theta_{z j} u_{x k} u_{y k} u_{z k} \theta_{x k} \theta_{y k} \theta_{z k}\right\}$.

Divide the displacement increment of the end of the member into elastic component and plastic component:

$\Delta \boldsymbol{u}=\Delta \boldsymbol{u}^{e}+\Delta \boldsymbol{u}^{p}$

Where $\Delta \boldsymbol{u}, \Delta \boldsymbol{u}^{e}$ and $\Delta \boldsymbol{u}^{p}$ are the increments of total displacement, elastic displacement and plastic displacement respectively.

Equilibrium equation of end section $j$ is:

$F_{i j}=\sum_{k=1}^{2} \sum_{s=1}^{6} K_{i j s k}^{e}\left(u_{s k}-u_{s k}^{p}\right)$

Where $F_{i j}$ is the component of section force in end section $j ; K_{i j s k}^{e}$ is the element of the element elastic stiffness matrix; $u_{s k}, u_{s k}^{p}$ are the component of the total displacement and the component of plastic displacement of end section $k$ respectively.

The plastic displacement, which is an accumulation of increments of the plastic displacement, is given by the following equation:

$\Delta \boldsymbol{u}_{k}^{p}=\Delta \lambda_{k} \frac{\partial \Phi_{k}}{\partial \boldsymbol{S}_{k}}$

Where $\Delta \boldsymbol{u}_{k}^{p}$ is the vector of plastic displacement increment of the end section $k ; \Delta \lambda_{k}$ is the proportion coefficient; $\boldsymbol{S}_{k}=\boldsymbol{F}_{k}-\boldsymbol{\alpha}_{k}, \boldsymbol{\alpha}_{k}$ is the vector of back stress of end section $k ; \Phi_{k}$ is the yield surface equation of end section $k$ which is given by the following equation:

$\Phi_{k}=\left(\frac{N_{x k}-\alpha_{N x k}}{N_{x u}}\right)^{2}+\left(\frac{M_{x k}-\alpha_{M x k}}{M_{x u}}\right)^{2}+\left(\frac{M_{y k}-\alpha_{M y k}}{M_{y u}}\right)^{2}+\left(\frac{M_{z k}-\alpha_{M z k}}{M_{z u}}\right)^{2}-1$ 
Where $N_{x u}, M_{x u}, M_{y u}$ and $M_{z u}$ represent the cross-sectional bearing capacities of the member at yield: the axial force and three moments, respectively; $N_{x k}$ is the axial force of the end section $k$; $M_{y k}$ and $M_{z k}$ are bending moments of the end section $k$ about the local 1- and 2-directions of the cross section of the member respectively; $M_{x k}$ is torsion of the end section $k ; \alpha_{N x k}, \alpha_{M x k}, \alpha_{M y k}$ and $\alpha_{M z k}$ are back stress components respectively.

The cross end section $k$ yields and the plastic hinge forms when $\Phi_{k} \geq 0$.

\subsection{Calculation Model of the Post-buckling Member}

Marshall model (Marshall, Gates, and Anagnostopoulos [9]), which is the mathematical description of the curve illustrated in Figure 4, is used to simulate the post-buckling member. The essence of Marshall model is the envelope of hysteresis loop of the inelastic circular tube (Figure 6). The envelope can be divided into 7 segments: (1) $A-F$ is the linear tension segment; (2) $F-F^{\prime}$ is the tension yielding segment; (3) $A-B$ is the linear compression segment; (4) $B-C$ is the first post buckling segment; (5) $C-D$ is the second post buckling segment; (6) $D-E$ is the first tension straightening segment; (7) $E-F$ is the second tension straightening segment. When reverse loading occurs at points on the boundary of the enclosed part of the envelope (such as point $B^{\prime}, C^{\prime}$ or $D^{\prime}$ ), the member exhibits damaged elastic behavior which is determined by drawing a line from the point on the envelope to the tension yield point (force value $P_{y}$ ). As long as the force and axial strain remain inside the enclosed part of the envelope, the force response is linear elastic with a modulus equal to the damaged elastic modulus. The axial force in the element is required to stay inside or on the envelope. When tension yielding occurs, the enclosed part of the envelope translates along the strain axis by an amount equal to the plastic strain. Coefficients in Figure 6 are defined as follows: $\gamma=0.02 ; \beta=0.02 ; \zeta=\min \left(1.0,5.8(t / D)^{0.7} / 0.95\right) ; \kappa=0.28$; $\alpha=0.03+0.004 L / D$, where $L$ is the length of the member; ultimate elastic axial force of the member is given by the following equation:

$P_{y}=0.95 \sigma_{s} A$

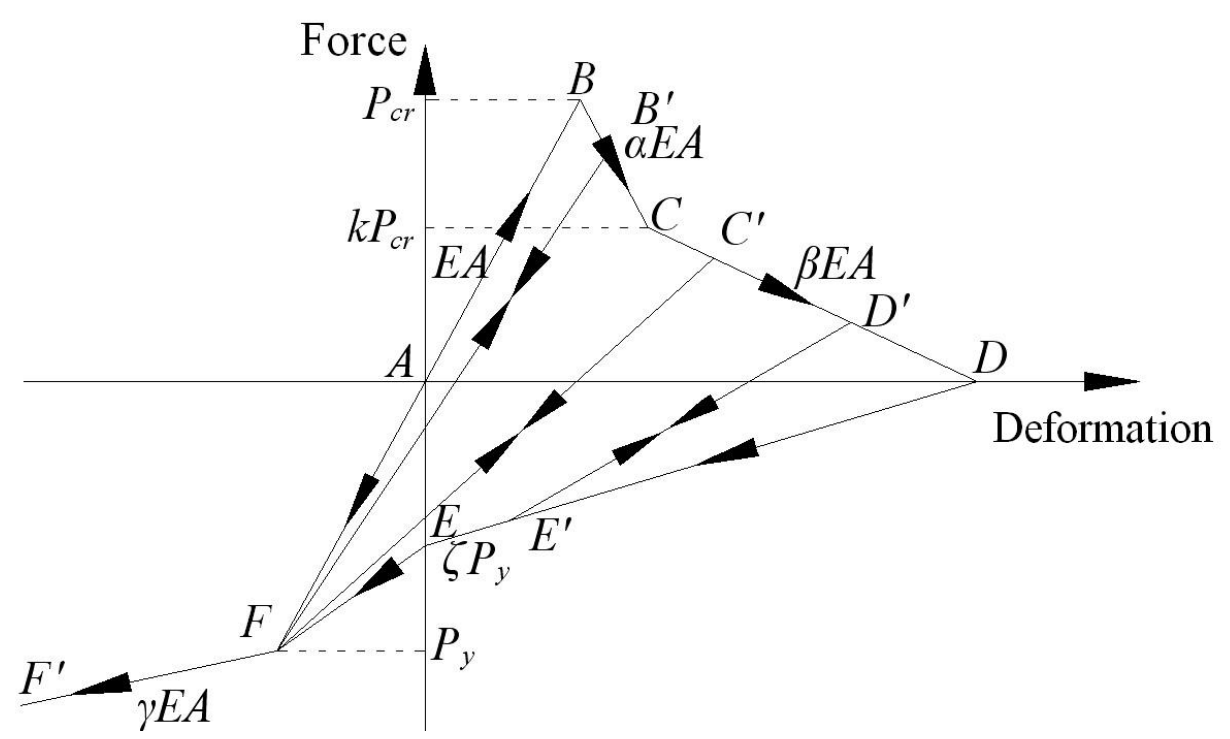

Figure 6. Marshall Model 
The structural member is firstly simulated by pre-buckling calculation model until Eq. 6 is met, and then Marshall model is used. If $\Phi \geq 0$ when Eq. 6 is med, the member performs buckling type I; otherwise it performs buckling type II . Plastic hinge of the end section of the member is simulated by pre-buckling calculation model, and that of the central section is simulated by Marshall model.

\section{CAUSES OF STRUCTURAL DYNAMIC DAMAGES}

Based on the refined member calculation model, numerical structure models of commonly used single layer latticed shells are created to analyze the causes of dynamic damages of the structures under earthquakes.

\subsection{Single Layer Spherical Latticed Shells}

Take a Kiewitt single layer spherical latticed shell with the span of $40 \mathrm{~m}$ and the rise-span ratio of $1 / 3$ as an example. The structure consists of circular tubes of $\Phi 114 \times 3.0, \Phi 127 \times 3.5$ and $\Phi 140 \times 4.5$ of steel Q235 (yield strength of $235 \mathrm{MPa}$ ), and bears surface load of $2.00 \mathrm{kN} / \mathrm{m}^{2}$ and seismic excitation of El Centro wave with peak acceleration of $620 \mathrm{gal}$ and duration of $12 \mathrm{~s}$.

Calculation results indicate that all of the 8 members in the structure central ring (Figure 7) buckle in $0.92 \mathrm{~s} \sim 0.93 \mathrm{~s}$ and perform buckling type II . Structure internal forces redistribute due to reductions of bearing capacities of the buckling members. Then the number of buckling member increases rapidly and the structural bearing capacity is weakened as a result. At $1.94 \mathrm{~s}$ the structural bearing capacity cannot keep balance with the seismic action, and the structure damages consequently. The structural strain energy increases suddenly with the strengthening of seismic action in $0.90 \mathrm{~s} \sim 0.92 \mathrm{~s}$ (Figure 8). The zoomed drawing shows that there are two slight fluctuations in the curve. They correspond to the member buckling processes which make the strain energy release. The strain energy releases greatly in $0.94 \mathrm{~s} \sim 0.95 \mathrm{~s}$, corresponding to the structural damage process.

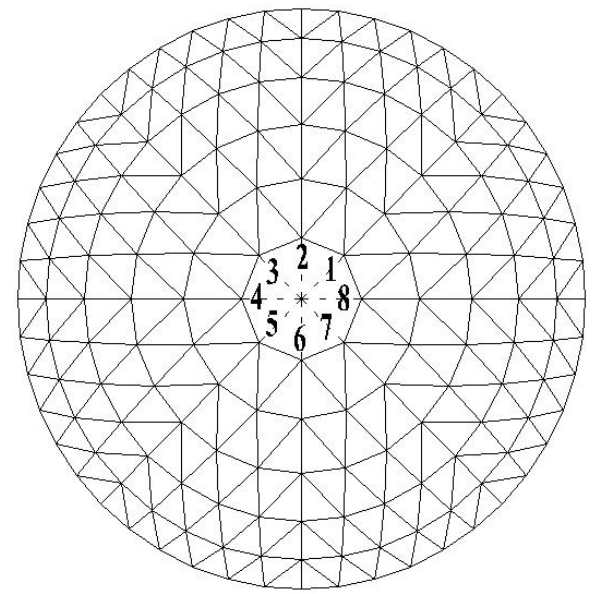

Figure 7. Buckling Members at 0.93s

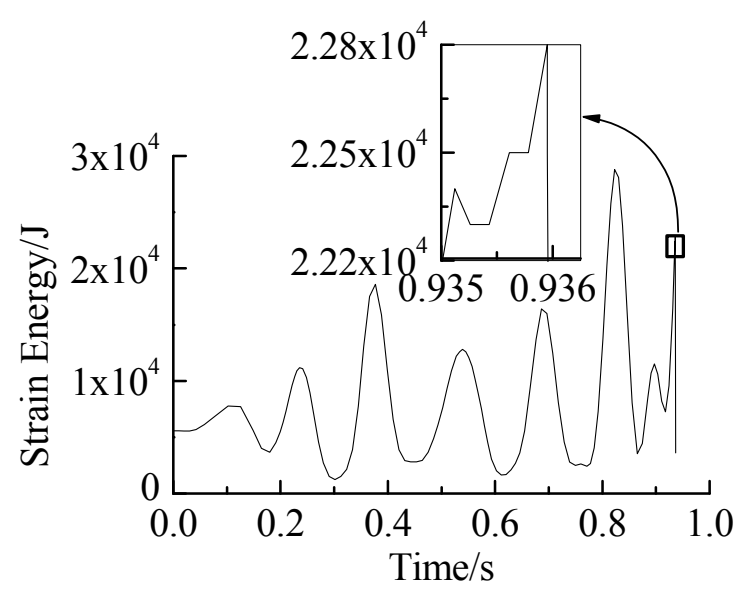

Figure 8. Time History of Structural Strain Energy

The peak acceleration of the seismic action is decreased to 350gal for a further investigation. Numerical results indicate that some structural members undergo buckle-straighten processes repeatedly and perform buckling type II all alike. The number of the buckling member changes continuously with the dynamic change of structural bearing capacity. The structure can hold yet with 32 buckling members at 1.69s (Figure 9) when the seismic action is not very strong. Thereafter some of the buckling members are straightened again. At $1.78 \mathrm{~s}$ the structure cannot hold 
the stronger seismic action and damages with 9 buckling members which are illustrated in Figure 10 .

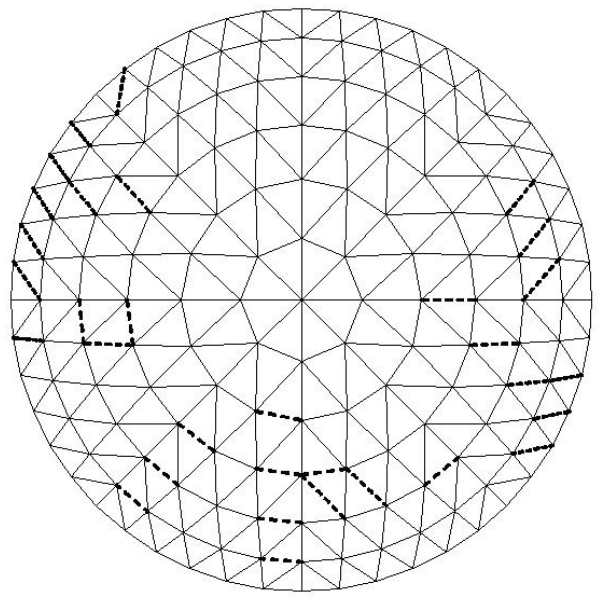

Figure 9. Buckling Members at 1.69s

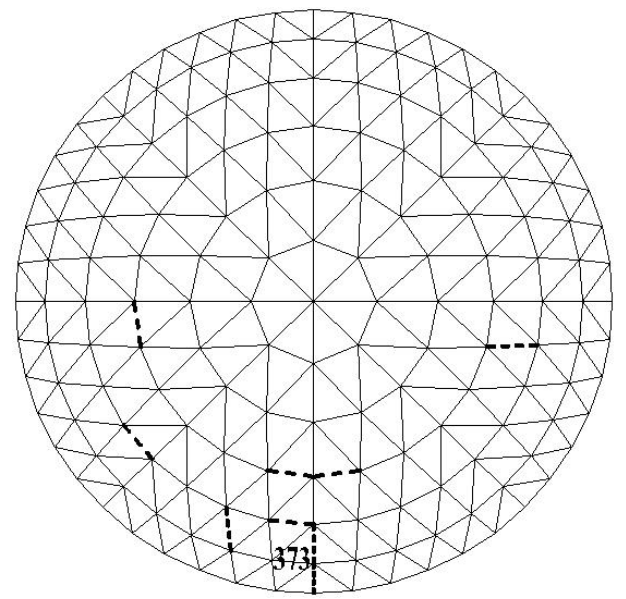

Figure 10. Buckling Members when Structure Damages

Comparing Figure 9 with Figure 10, it can be seen that the structural damage is determined by the dynamic balance between the structural bearing capacity and the seismic action rather than the number of buckling members.

Axial fore time history of member 373 (marked in Figure 10) is illustrated in Figure 11. The member buckles when axial force reaches its limit buckling axial force of $164 \mathrm{kN}$. After the member buckles, the axial force turns small rapidly. It can be seen that the member buckles twice.

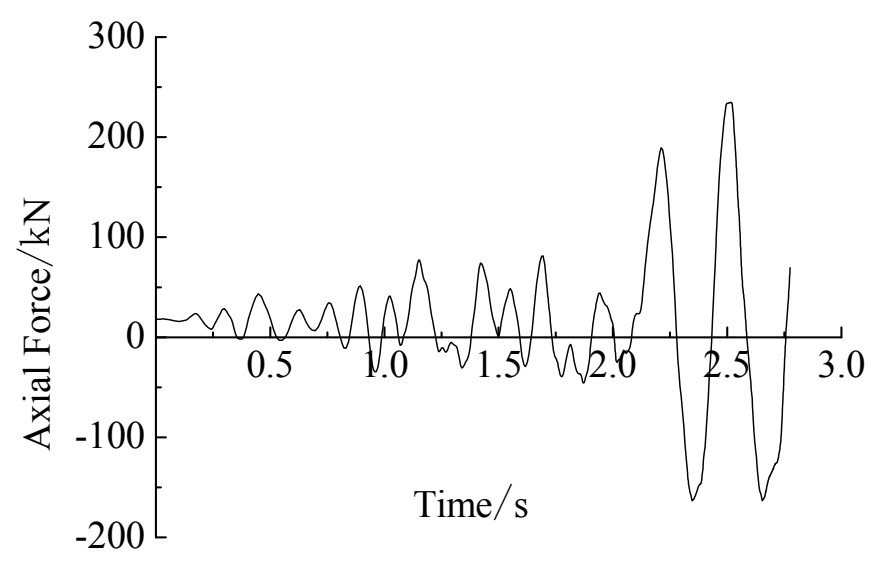

Figure 11. Time History of Axial Force of Member 373

The structural members are simulated by the general beam element of general finite element analysis program, and the axial fore time history of member 373 is illustrated in Figure 12. It can be seen that the maximum axial fore in member 373 reaches $246.14 \mathrm{kN}$ which has far surpassed the limit buckling axial force value, so the bearing capacities of the members are overestimated. The structural dynamic bearing capacity can keep balance with the seismic action in the entire $12 \mathrm{~s}$, and the error conclusion that the structure does not damage may be made consequently. 


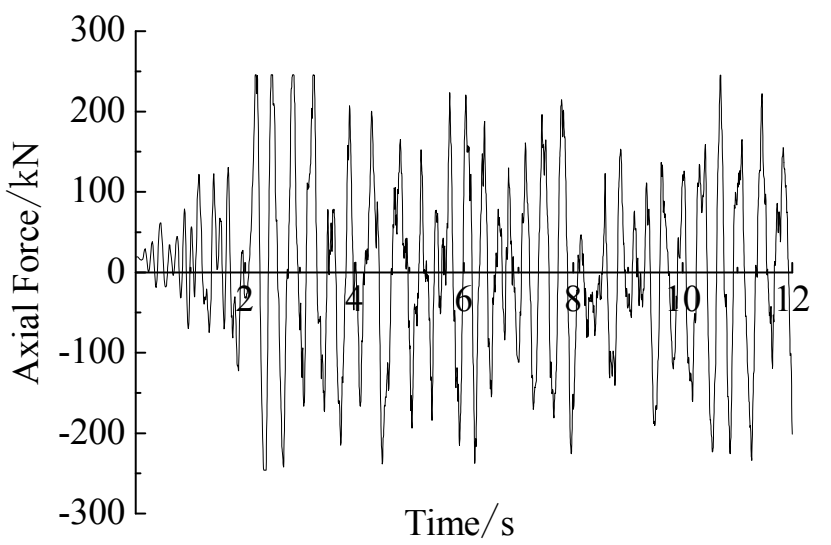

Figure 12. Axial Fore Time Histories of Member 373 Calculated by FEM Program

Numerical models of five kinds of commonly used single layer spherical latticed shells with the uniform span of $40 \mathrm{~m}$ and different rise-span ratios of 1/4, 1/5, 1/6 and 1/7 are created respectively for the parameter analysis. Seismic excitation of El Centro wave with peak acceleration of 900gal and duration of $12 \mathrm{~s}$ is applied. Numbers of buckling members and plastic hinges in the end sections of the members when structures damage are presented in Table 1.

Table 1. Numbers of Buckling Members and Plastic Hinges when Single Layer Spherical Latticed Shells Damage

\begin{tabular}{|c|c|c|c|c|c|c|}
\hline \multirow{2}{*}{$\begin{array}{l}\text { Rise-span } \\
\text { ratio }\end{array}$} & \multirow{2}{*}{$\begin{array}{l}\text { Buckling members/ } \\
\text { Plastic hinges }\end{array}$} & \multicolumn{5}{|c|}{ Types of single layer spherical latticed shell } \\
\hline & & Lamella grid & Schwedler & Ribbed type & $\begin{array}{l}\text { Three-way } \\
\text { grid }\end{array}$ & Kiewitt \\
\hline \multirow{2}{*}{$1 / 4$} & Buckling members & 25 & 27 & 25 & 29 & 32 \\
\hline & Plastic hinges & 0 & 0 & 0 & 0 & 0 \\
\hline \multirow{2}{*}{$1 / 5$} & Buckling members & 21 & 22 & 24 & 28 & 29 \\
\hline & Plastic hinges & 0 & 0 & 0 & 0 & 0 \\
\hline \multirow{2}{*}{$1 / 6$} & Buckling members & 18 & 18 & 20 & 22 & 22 \\
\hline & Plastic hinges & 0 & 0 & 0 & 0 & 0 \\
\hline \multirow{2}{*}{$1 / 7$} & Buckling members & 17 & 16 & 17 & 18 & 20 \\
\hline & Plastic hinges & 1 & 2 & 0 & 0 & 0 \\
\hline
\end{tabular}

Table 1 shows that the members of single layer spherical latticed shells under earthquake perform buckling type II mainly. Structures of bigger rise-span ratios have more buckling members when they damage. Plastic hinges of the end sections of the members are quite rare in the structures. Except for the two structures of lamella grid and Schwedler with the rise-span ratio of 1/7, there are no plastic hinges in the end sections of the members in other single layer spherical latticed shells when they damage. Therefore dynamic damages of single layer spherical latticed shells are caused by buckling members which weaken the structural bearing capacity.

\subsection{Single Layer Cylindrical Latticed Shells}

Take a three-way grid single layer cylindrical latticed shell with the span of $15 \mathrm{~m}$, the length of $21 \mathrm{~m}$ and the rise-span ratio of $1 / 2$ as an example. The structure consists of circular tubes of $\Phi 114 \times 3.0$, $\Phi 127 \times 3.5$ and $\Phi 140 \times 4.5$ of steel Q235, and bears surface load of $1.00 \mathrm{kN} / \mathrm{m}^{2}$ and seismic excitation of El Centro wave with peak acceleration of 620gal and duration of $12 \mathrm{~s}$. 
Numerical results indicate that it is the end section of the member of single layer cylindrical latticed shell yields firstly. Along with the increment of the load, plastic hinges form in the end sections of some members which cannot bear bigger bending moments and shear forces while the deformations become larger rapidly. This causes the redistribution of the structural internal forces, and more plastic hinges form in the end sections of the conjoint members. The concentrative plastic hinges in the end sections of conjoint members may make parts of the structure turn to mechanism. At $6.04 \mathrm{~s} 43$ plastic hinges form in the end sections of the members as illustrated in Figure 13 (black dots represent plastic hinges, and hollow rings represent plastic zones where plastic hinges have not formed yet.). If seismic action weakens afterward, the plastic hinges could disappear and residual deformations remain. The number of plastic hinges and positions where they form change continuously and it makes the consequent change of the structural bearing capacity. At $6.13 \mathrm{~s}$ the structure cannot hold the stronger seismic action and damages with 26 plastic hinges in the end sections of the members which are illustrated in Figure 14.

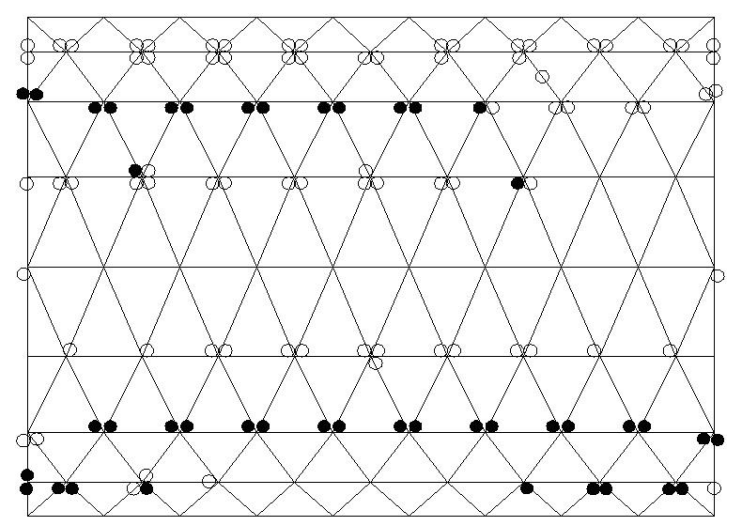

Figure 13. Plastic Hinges at 6.04s

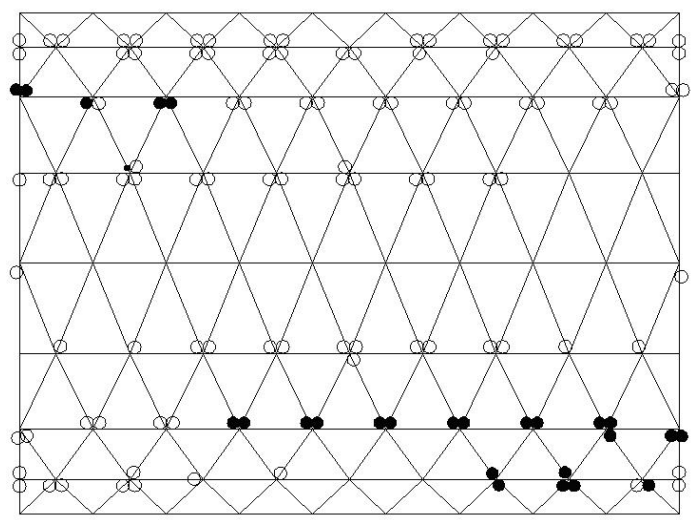

Figure 14. Plastic Hinges when Structure Damages

Comparing Figure 13 with Figure 14, it can be seen that the structural damage is determined by the dynamic balance between the structural bearing capacity and the seismic action rather than the number of plastic hinges.

Numerical models of four kinds of commonly used single layer cylindrical latticed shells with the uniform span of $15 \mathrm{~m}$, the uniform length of $21 \mathrm{~m}$, and different rise-span ratios of $1 / 2,1 / 3,1 / 4$ and $1 / 5$ are created respectively for the parameter analysis. Seismic excitation of El Centro wave with peak acceleration of $900 \mathrm{gal}$ and duration of $12 \mathrm{~s}$ is applied. Numbers of buckling members and plastic hinges of the end sections of the members when structures damage are presented in Table 2.

Table 2. Numbers of Buckling Members and Plastic Hinges when Single Layer Cylindrical Latticed Shells Damage

\begin{tabular}{cccccc}
\hline \multirow{2}{*}{$\begin{array}{c}\text { Rise-span } \\
\text { ratio }\end{array}$} & Buckling members/ $/ 4$ & \multicolumn{3}{c}{ Types of single layer cylindrical latticed shell } \\
\cline { 3 - 6 } $1 / 2$ & Plastic hinges & $\begin{array}{c}\text { Orthogonal grid } \\
\text { with one bracing }\end{array}$ & $\begin{array}{c}\text { Orthogonal grid } \\
\text { with two bracings }\end{array}$ & $\begin{array}{c}\text { Lamella } \\
\text { grid }\end{array}$ & $\begin{array}{c}\text { Three-way } \\
\text { grid }\end{array}$ \\
\hline \multirow{2}{*}{$1 / 3$} & Plastic hinges & 34 & 0 & 0 & 1 \\
& Buckling members & 0 & 25 & 16 & 24 \\
& Plastic hinges & 37 & 0 & 0 & 0 \\
$1 / 4$ & Buckling members & 0 & 26 & 21 & 29 \\
& Plastic hinges & 42 & 0 & 0 & 0 \\
$1 / 5$ & Buckling members & 0 & 30 & 22 & 30 \\
& Plastic hinges & 43 & 0 & 0 & 0 \\
\hline
\end{tabular}


Table 2 shows that the members of single layer cylindrical latticed shells under earthquake perform buckling type I mainly. Structures of smaller rise-span ratios have more plastic hinges in the end sections of the members when they damage. Buckling members are quite rare in the structures. Except for the two structures of orthogonal grid with one bracing and three-way grid with the rise-span ratio of $1 / 2$, there are no buckling members in other types of the single layer cylindrical latticed shells when they damage. Therefore dynamic damages of single layer cylindrical latticed shells are caused by concentrative plastic hinges in the end sections of some members which make parts of the structure turn to mechanisms.

\subsection{Single Layer Elliptic Paraboloid Latticed Shells}

Take an orthogonal grid with one bracing single layer elliptic paraboloid latticed shell with the span of $30 \mathrm{~m}$, the length of $48 \mathrm{~m}$ and the rise-span ratio of $1 / 3$ as an example. The structure consists of circular tubes of $\Phi 114 \times 4.0, \Phi 121 \times 4.0, \Phi 146 \times 6.0$ and $\Phi 152 \times 6.0$ of steel Q235, and bears surface load of $1.00 \mathrm{kN} / \mathrm{m}^{2}$ and seismic excitation of El Centro wave with peak acceleration of $620 \mathrm{gal}$ and duration of $12 \mathrm{~s}$.

Numerical results indicate that buckling members and plastic hinges in the end sections of the members appear together in large numbers and the members of the single layer elliptic paraboloid latticed shell subjected to seismic excitations perform both the buckling type I and buckling type II. Time histories of the numbers of buckling members and plastic hinges in the end sections are illustrated in Figure 15 and Figure 16 respectively. The recurrent buckle-straighten processes of the members weaken the structural bearing capacity, and the recurrent form-disappear processes of the plastic hinges of the end sections make parts of the structure turn to mechanisms. The structure damages as a combined action of the two causes mentioned above. Buckling members and plastic hinges in the end sections when the structure damages are illustrated in Figure 17.
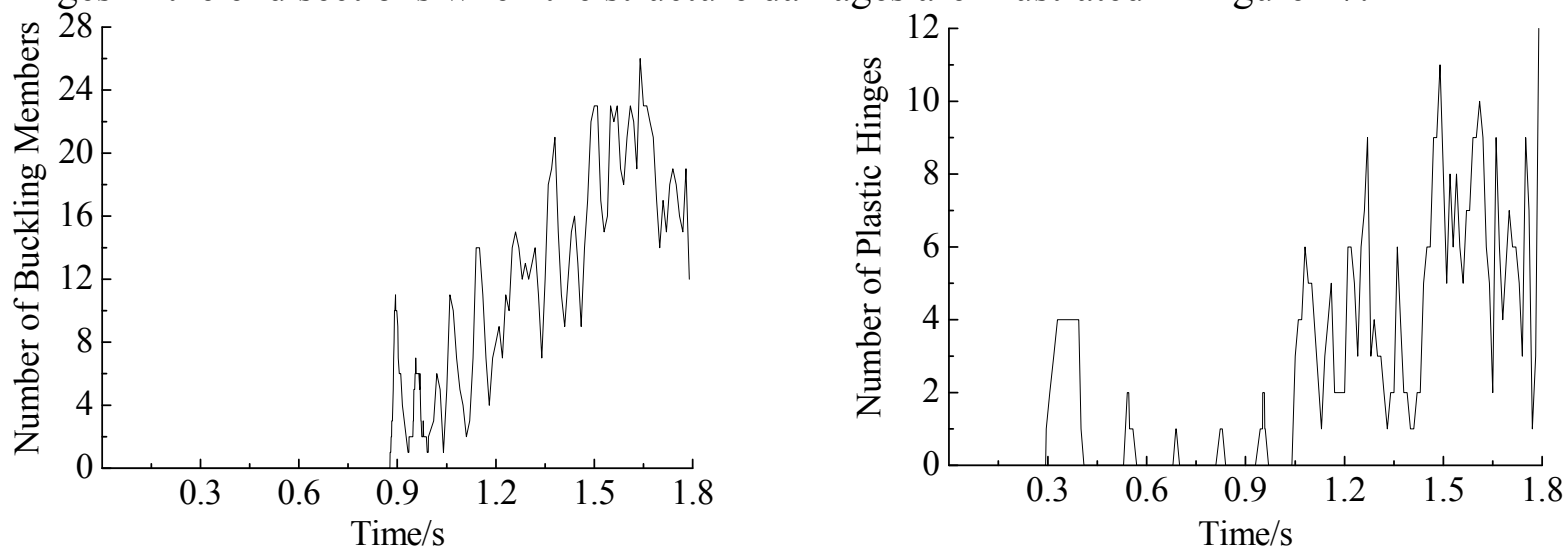

Figure 15. Number Change of Buckling Members Figure 16. Number Change of Plastic Hinges

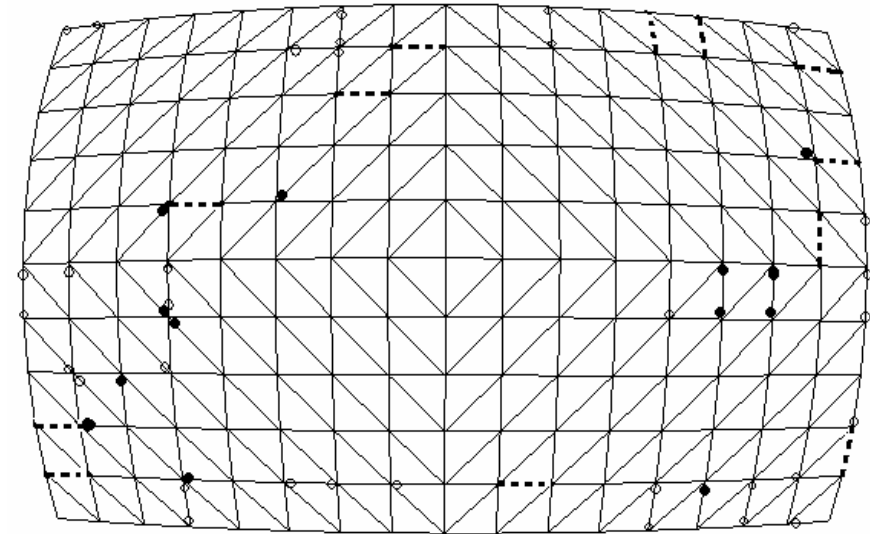

Figure 17. Buckling Members and Plastic Hinges when Structure Damages 
Numerical models of two kinds of commonly used single layer elliptic paraboloid latticed shells with the uniform span of $30 \mathrm{~m}$, the uniform length of $48 \mathrm{~m}$, and different rise-span ratios of $1 / 3,1 / 4$, $1 / 5$ and $1 / 6$ are created respectively for the parameter analysis. Seismic excitation of El Centro wave with peak acceleration of $900 \mathrm{gal}$ and duration of $12 \mathrm{~s}$ is applied. Numbers of buckling members and plastic hinges in the end sections of the members when structures damage are presented in Table 3.

Table 3. Numbers of Buckling Members and Plastic Hinges when

Single Layer Elliptic Paraboloid Latticed Shells Damage

\begin{tabular}{cccc}
\hline \multirow{2}{*}{$\begin{array}{c}\text { Rise-span } \\
\text { ratio }\end{array}$} & Buckling members/ & \multicolumn{2}{c}{ Types of single layer elliptic paraboloid latticed shell } \\
\cline { 3 - 4 } $1 / 3$ & Plastic hinges & Orthogonal grid with one bracing & Three-way grid \\
\hline \multirow{2}{*}{$1 / 4$} & Buckling members & 28 & 26 \\
& Plastic hinges & 15 & 25 \\
& Buckling members & 25 & 26 \\
& Plastic hinges & 16 & 29 \\
& Buckling members & 22 & 22 \\
\multirow{2}{*}{$1 / 6$} & Plastic hinges & 20 & 30 \\
& Buckling members & 20 & 15 \\
& Plastic hinges & 23 & 33 \\
\hline
\end{tabular}

Table 3 shows that the members of the single layer elliptic paraboloid latticed shell under earthquake perform both the buckling type I and buckling type II. Structures of bigger rise-span ratios have more buckling members and fewer plastic hinges in the end sections of the members when they damage. Dynamic damages of single layer elliptic paraboloid latticed shells are caused by buckling members which weaken the structural bearing capacity and concentrative plastic hinges in the end sections of some members which make parts of the structure turn to mechanisms together.

\section{CONCLUSIONS}

The dynamic damages causes of single layer latticed shells subjected to seismic excitations based on refined member calculation model are studied in this paper. Based on the analysis, some conclusions can be drawn as follows:

(1) If the members of single layer latticed shells are simulated by general beam element of general finite element analysis program, the elastic-plastic element stiffness matrix of the member is not accurate and the buckling of the member cannot be simulated as well. By this method, the structural bearing capacity will be overestimated.

(2) Two buckling types of the member of single layer latticed shells are presented. Buckling type I: the plastic hinge forms in the end section of the member, and the member slenderness ratio gets bigger. This may cause buckling of the member. Buckling type II : the member bears compression axial force plenty big and the buckling critical condition is met without the plastic hinge forming in the end section.

(3) The pre-buckling and post-buckling mechanical behaviors of the structural member are simulated by different models, and the refined member calculation model is established. By this model the second order effect of the member is considered, and the possible buckle-straighten processes of the members and the form-disappear processes of the structure under earthquake can be simulated.

(4) The dynamic damages of the structures under earthquake are determined by the dynamic balance between the structural bearing capacity and the seismic action rather than the 
number of buckling members or the plastic hinges.

(5) Dynamic damage causes of single layer latticed shells vary with different structural types: dynamic damages of single layer spherical latticed shells are caused by buckling members which weaken the structural bearing capacity; dynamic damages of single layer cylindrical latticed shells are caused by concentrative plastic hinges in the end sections of some members which make parts of the structure turn to mechanisms; dynamic damages of single layer elliptic paraboloid latticed shells are caused by combined actions of the two causes mentioned above.

\section{ACKNOWLEDGEMENTS}

Financial supports from National Natural Science Foundation of China (Grant No. 90715034 and 50978181), Program for New Century Excellent Talents in University, Ministry of Education of China (Grant No. NCET-06-0229), and Program for Applied Foundation and Advanced Technology, Tianjin, China (Grant No. 09JCZDJC25200) are gratefully acknowledged.

\section{REFERENCES}

[1] Kani, I.M. and Heidari, A., "Automatic Two-Stage Calculation of Bifurcation Path of Perfect Shallow Reticulated Domes", Journal of Structural Engineering, 2007, Vol. 133, No. 2, pp. 185-194.

[2] Yamada, S., Takeuchi, A., Tada, Y., et al., "Imperfection-Sensitive Overall Buckling of Single- Layer Lattice Domes”, Journal of Engineering Mechanics, 2001, Vol. 127, No. 4, pp. 382-386.

[3] Hearn, G. and Adams, E., "Shape Selection for Lattice Structures", Journal of Structural Engineering, 2006, Vol. 132, No. 11, pp. 1713-1720.

[4] Morris, N.F., "Effect of Imperfections on Lattice Shells", Journal of Structural Engineering, 2007, Vol. 117, No. 6, pp. 1796-1814.

[5] Kani, I.M. and McConnel, R.E., "Collapse of Shallow Lattice Domes", Journal of Structural Engineering, 1987, Vol. 113, No. 8, pp. 1806-1819.

[6] Fan, F., "Report for Refined Research Advances on Disastrous Damages of Long Span Spatial Structures Subjected to Severe Earthquakes", Seminar on Major Program of National Natural Science Foundation of China. Dalian, China, 2009.

[7] Chen, W.F. and Sugimoto, H., "Stability and Strength of Fabricated Tubular Columns Used in Offshore Structures", Research Report of Purdue University, West Lafayette, U.S.A., 1984.

[8] International Standards Organization, "Steel Structures: Materials and Design", Specification for the Design and Construction of Steel Structures (ISO 10721-1), 1997.

[9] Marshall, P.W., Gates, W.E., and Anagnostopoulos, S., "Inelastic Dynamic Analysis of Tubular Offshore Structures", Proceedings of Ninth Annual Offshore Technology Conference, Houston, U.S.A., 1977, pp. 235-246. 